International Journal of Pure and Applied Mathematics

Volume 100 No. 3 2015, 371-378

ISSN: 1311-8080 (printed version); ISSN: 1314-3395 (on-line version)

url: http://www.ijpam.eu

doi: http://dx.doi.org/10.12732/ijpam.v100i3.3

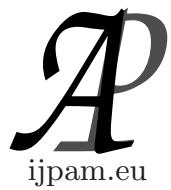

\title{
NEW MIXED FINITE VOLUME SPACES FOR ELLIPTIC PROBLEMS
}

\author{
JiHyun Kim \\ Department of Mathematics \\ Hannam University \\ 133 Ojeong-Dong, Daedeok-Gu, Daejeon 306-791, REPUBLIC OF KOREA
}

\begin{abstract}
We introduce a new family of mixed finite volume spaces of higher order for second order elliptic problems on rectangular grids. We also show that these volume methods are equivalent to the nonconforming finite element methods used to define them.
\end{abstract}

AMS Subject Classification: 65 N15, 65N30, 35J60

Key Words: mixed finite element methods, mixed finite volume methods, nonconforming finite element methods

\section{Introduction}

Mixed finite element methods for elliptic problems have been the subject of extensive research(see [1], [2], [3], [4], [5], [6]). The main idea of mixed finite element methods is to introduce the Darcy velocity as a new variable and write the equation into a system of differential equations. By discretizing this system, we can compute velocity and pressure together. However, the resulting mixed system has many more variables and gives rise to a saddle point problem. Thus there were some restrictions to use in industry. For the lowest order mixed methods, it is well known that there exist equivalent nonconforming finite element methods[7], [8]. For higher order cases, there exist some equivalent nonconforming finite element spaces. But this methods involve projections into

Received: October 1, 2014

(c) 2015 Academic Publications, Ltd. url: www.acadpubl.eu 
the vector part of the mixed finite element spaces, which are not easy to handle. To overcome this difficulty, a finite volume type of mixed method and its variants were introduced(see [9], [10], [11], [12]). The idea is to integrate the system over each element with weight functions. Assuming the velocity variable in the Raviart-Thomas space, and the scalar variables lies in $Q_{1}$ nonconforming finite element space, they show the equivalence between the mixed finite element method and the nonconforming finite element method. Thus the solution of the mixed finite volume methods can be obtained through these nonconforming finite element method.

In this paper, we define new finite volume type of mixed methods with judiciously chosen test spaces and show that they are equivalent to some nonconforming finite element methods used to define them.

The organization of this paper is as follows: In the next section, we start with a problem description and presentation of the basic materials necessary to study mixed finite volume methods. In section 3, we define our new family of finite volume mixed method. Under a series of hypotheses, we show these methods are equivalent to some nonconforming finite element methods.

\section{Mixed Finite Volume Methods}

In this section, we introduce mixed finite volume methods and related nonconforming finite element methods. Let $\Omega$ be a boundary polygonal domain in $\mathbb{R}^{2}$ with the boundary $\partial \Omega$. We consider the following second order elliptic boundary value problem:

$$
\left\{\begin{aligned}
-\operatorname{div}(\kappa \nabla p) & =f, & & \text { in } \Omega, \\
p & =0, & & \text { on } \partial \Omega,
\end{aligned}\right.
$$

where $\kappa=\kappa(\mathbf{x})$ is a symmetric and uniformly positive definite matrix, i.e., there exist two positive constants $c_{1}$ and $c_{2}$ such that

$$
c_{1} \xi^{T} \xi \leq \xi^{T} \kappa(\mathbf{x}) \xi \leq c_{2} \xi^{T} \xi, \quad \forall \xi \in \mathbb{R}^{2}, \mathbf{x} \in \bar{\Omega} .
$$

For the discussion of higher order methods, we shall require $f \in H^{k}(\Omega)$ for some integer $k \geq 0$. Let us introduce a vector variable $\mathbf{u}=-\kappa \nabla p$ and reformulate the problem (1) in the following mixed form:

$$
\left\{\begin{array}{rlrl}
\mathbf{u}+\kappa \nabla p & =0, & & \text { in } \Omega, \\
\operatorname{div} \mathbf{u}=f, & & \text { in } \Omega, \\
p & =0, & & \text { on } \partial \Omega .
\end{array}\right.
$$


First, we introduce some function spaces. For any domain $\Omega$, we let $L^{2}(\Omega)$ be the set of all square integrable functions on $\Omega$ equipped with the usual inner product $(\cdot, \cdot)_{\Omega}$. Let $H^{k}(\Omega)=W^{k, 2}(\Omega)$ be the Sobolev spaces of order $k \geq 0$ with obvious norms and $\mathbf{H}^{k}(\Omega)$ be the space of vectors each of whose component lies in $H^{k}(\Omega)$. For both of the spaces $H^{k}(\Omega)$ and $\mathbf{H}^{k}(\Omega)$, we denote the norms(semi-norms) by $\|\cdot\|_{k, \Omega}\left(|\cdot|_{k, \Omega}\right)$ or $\|\cdot\|_{k}\left(|\cdot|_{k}\right)$ when no confusion arises. Throughout this paper, we assume that the solution $(\mathbf{u}, p)$ of $(2)$ satisfies $\mathbf{u} \in \mathbf{H}^{k+1}(\Omega), p \in H^{k+2}(\Omega)$, and there exists some constant $C>0$ such that

$$
\|\mathbf{u}\|_{k+1}+\|p\|_{k+2} \leq C\|f\|_{k} .
$$

Also, let $\mathbf{V}=H(\operatorname{div}, \Omega)=\left\{\mathbf{v} \in\left(L^{2}(\Omega)\right)^{2} \mid \operatorname{div} \mathbf{v} \in L^{2}(\Omega)\right\}$ and $W=L^{2}(\Omega)$. Then we have the following variational form for $(2)$ :

$$
\left\{\begin{array}{rlrl}
\left(\kappa^{-1} \mathbf{u}, \mathbf{v}\right)-(p, \operatorname{div} \mathbf{v}) & =0, & \forall \mathbf{v} \in \mathbf{V}, \\
(\operatorname{div} \mathbf{u}, q) & =(f, q), \quad \forall q \in W .
\end{array}\right.
$$

This problem has a unique solution pair $(\mathbf{u}, p) \in \mathbf{V} \times W$. Now we consider the finite element method. From now on, we assume the domain can be partitioned into rectangles. Assume that we have some finite element subspaces $\mathbf{V}_{h} \subset \mathbf{V}$ and $W_{h} \subset W$ based on these grids. Then the corresponding finite dimensional approximation $\left(\mathbf{u}_{h}, p_{h}\right) \in \mathbf{V}_{h} \times W_{h}$ is defined as the solution of the following equations:

$$
\left\{\begin{aligned}
\left(\kappa^{-1} \mathbf{u}_{h}, \mathbf{v}_{h}\right)-\left(p_{h}, \operatorname{div} \mathbf{v}_{h}\right) & =0, & & \forall \mathbf{v}_{h} \in \mathbf{V}_{h} \\
\left(\operatorname{div} \mathbf{u}_{h}, q_{h}\right) & =\left(f, q_{h}\right), & & \forall q_{h} \in W_{h}
\end{aligned}\right.
$$

To explain a higher order mixed finite volume method, we need some hypotheses[12]. Let $\mathbf{V}_{h}$ be the vector part of any mixed finite element space and let $Q$ be a rectangular element.

$(\mathbf{H} 1)$ There is a nonconforming finite element space $N_{h}$ of order $k$ associated with the same triangulation such that for $\chi \in N_{h}$ the moments up to $k$

$$
\int_{e} \chi q d s, q \in P_{k}(e), \quad \text { for any edge } e \text { of } \mathrm{Q},
$$

are continuous across interior edges,

$$
\int_{e} \chi q d s, \quad q \in P_{k}(e), \quad \text { if edge } e \text { is part of } \partial \Omega,
$$

and the interior degrees of freedom are given by

$$
\int_{Q} \chi \phi d x, \phi \in N_{h}^{i}(Q)
$$


for some space $N_{h}^{i}(Q)$ of polynomials.

(H2) The space $N_{h}(Q)$ has the approximation property

$$
\inf _{v_{\mathrm{h}} \in N_{\mathrm{h}}(Q)}\left(\left\|v-v_{h}\right\|_{0}+h\left|v-v_{h}\right|_{1}\right) \leq C h^{k+2}\|v\|_{k+2},
$$

whenever $v \in H^{k+2}(Q)$.

$(\mathbf{H} 3)$ Let $U_{h}(Q)=\operatorname{div} \mathbf{V}_{h}(Q)$ and $\boldsymbol{\Psi}_{h}(Q)$ be the interior degrees of freedom of $\mathbf{V}_{h}(Q)$. Then the space $U_{h}(Q)$ satisfies

$$
\nabla U_{h}(Q) \subset \boldsymbol{\Psi}_{h}(Q),
$$

and

$$
U_{h}(Q) \subset N_{h}(Q) .
$$

$(\mathbf{H} 4)$ There is a space of polynomial $\mathbf{V}_{h}^{s}(Q)$ such that

$$
\mathbf{V}_{h}^{s}(Q) \oplus \nabla U_{h}(Q) \supset \boldsymbol{\Psi}_{h}(Q),
$$

and

$$
\operatorname{dim} \mathbf{V}_{h}^{s}(Q)=\operatorname{dim} \boldsymbol{\Psi}_{h}(Q)-\operatorname{dim} U_{h}(Q)+1 .
$$

Now, we present a higher order mixed finite volume method. Find $\left(\mathbf{u}_{h}, p_{h}\right) \in$ $\mathbf{V}_{h}(Q) \times N_{h}(Q)$ which satisfies on every element $Q \in \mathscr{T}_{h}$

$$
\begin{aligned}
& \int_{Q}\left(\mathbf{u}_{h}+\kappa \nabla p_{h}\right) \cdot \nabla \chi d x=0, \quad \forall \chi \in N_{h}(Q), \\
& \int_{Q}\left(\mathbf{u}_{h}+\kappa \nabla p_{h}\right) \cdot \mathbf{v} d x=0, \quad \forall \mathbf{v} \in \mathbf{V}_{h}^{s}(Q), \\
& \int_{Q} \operatorname{div} \mathbf{u}_{h} \phi d x=\int_{Q} f \phi d x, \quad \forall \phi \in U_{h}(Q) .
\end{aligned}
$$

From (13), we know that

$$
\operatorname{div} \mathbf{u}_{h}=P_{U_{\mathrm{h}}} f,
$$

where $P_{U_{\mathrm{h}}}$ is an $L^{2}$-projection onto $U_{h}$. If $\chi \in N_{h}(Q)$, we have

$$
\begin{aligned}
\sum_{Q} \int_{Q} \kappa \nabla p_{h} \cdot \nabla \chi d x & =-\sum_{Q} \int_{Q} \mathbf{u}_{h} \nabla \chi d x \\
& =-\sum_{Q} \int_{\partial Q} \mathbf{u}_{h} \cdot \mathbf{n} d s+\sum_{Q} \int_{Q} \operatorname{div} \mathbf{u}_{h} \chi d x
\end{aligned}
$$


Since $\chi$ has continuous moments up to degree $k$ across internal edges and has vanishing moments on $\partial \Omega$,

$$
\sum_{Q} \int_{Q} \kappa \nabla p_{h} \cdot \nabla \chi d x=\int_{\Omega}\left(P_{U_{\mathrm{h}}} f\right) \chi d x
$$

Since

$$
\int_{\Omega}\left(P_{U_{\mathrm{h}}} f\right) \chi d x=\int_{\Omega} f\left(P_{U_{\mathrm{h}}} \chi\right) d x
$$

we can obtain the following equation:

$$
\sum_{Q} \int_{Q} \kappa \nabla p_{h} \cdot \nabla \chi d x=\int_{\Omega} f\left(P_{U_{\mathrm{h}}} \chi\right) d x, \text { for } \chi \in N_{h}(Q) .
$$

From (15), we know that the mixed finite volume method is equivalent to a standard nonconforming finite element method, except that $L^{2}$-projections are used on the lower order terms. Thus to implement (11)-(13), it suffices to solve the equivalent system (15).

\section{New mixed finite volume spaces for rectangular element}

For simplicity, we let $Q=[-1,1] \times[-1,1]$. Let $\mathbf{V}_{h}(Q)$ be the following element of order $k$ :

$$
\mathbf{V}_{h}(Q)=P_{k+1, k} \times P_{k, k+1},
$$

where the two elements $\left(x^{k+1} y^{k}, 0\right)^{T}$ and $\left(0, x^{k} y^{k+1}\right)^{T}$ are replaced by the single element $\left(x^{k+1} y^{k},-x^{k} y^{k+1}\right)^{T}$. To define the degrees of freedom, we first let

$$
\mathbf{\Psi}_{h}(Q)=P_{k-1, k} \times P_{k, k-1},
$$

where $\left(x^{k-1} y^{k}, 0\right)^{T}$ and $\left(0, x^{k} y^{k-1}\right)^{T}$ are replaced by the element $\left(x^{k-1} y^{k},-x^{k}\right.$ $\left.y^{k-1}\right)^{T}$. Then, for any $\mathbf{u} \in \mathbf{V}_{h}(Q)$, we have the following degrees of freedom:

$$
\begin{aligned}
& \int_{e} \mathbf{u}_{h} \times \mathbf{n} q d s, \quad q \in P_{k}(e), \quad \text { for each edge } e \text { of } Q, \\
& \int_{Q} \mathbf{u}_{h} \cdot \phi d \mathbf{x}, \quad \phi \in \mathbf{\Psi}_{h}(Q) .
\end{aligned}
$$

Now consider a new nonconforming finite element space:

$$
N_{h}(Q)=R_{h}(Q) \oplus X^{k}(Q) \oplus Y^{k}(Q),
$$


where $R_{h}(Q)$ be the space of all polynomials in each variable up to degree $k$ except a constant multiple of the term $x^{k} y^{k}$. And

$$
\begin{aligned}
& X^{k}(Q)=\left\{\sum_{i=0}^{k}\left[a_{i, 0} l_{k+1}(x)+a_{i, 1} l_{k+2}(x)\right] l_{i}(y)\right\}, \\
& Y^{k}(Q)=\left\{\sum_{i=0}^{k}\left[b_{i, 0} l_{k+1}(y)+b_{i, 1} l_{k+2}(y)\right] l_{i}(x)\right\},
\end{aligned}
$$

where $l_{i}$ is the Legendre polynomial on $[-1,1] \times[-1,1]$. Then the function in $N_{h}(Q)$ is completely determined by (5) and (7) with the choice of $N_{h}^{i}(Q)=$ $R_{h}(Q)$. Hence $(\mathbf{H} 1)$ holds. Since $N_{h}(Q) \supset P_{k+1}(Q),(\mathbf{H} 2)$ holds. Also, (H3) is trivial, since $U_{h}(Q)=R_{h}(Q)$. We choose

$$
\mathbf{V}_{h}^{s}(Q)=P_{k-1, k} \times P_{k, k-1} \cap \nabla P_{k, k}^{\perp} .
$$

Since $\nabla P_{k, k} \subset P_{k-1, k} \times P_{k, k-1}$, the dimension of $\mathbf{V}_{h}^{s}(Q)$ is $\operatorname{dim} \mathbf{V}_{h}^{s}(Q)=\operatorname{dim}\left(P_{k-1, k} \times P_{k, k-1}\right)-\operatorname{dim}\left(\nabla P_{k, k}\right)=2 k(k+1)-(k+1)^{2}+1=k^{2}$. To check (H4), we first see that

$$
\mathbf{V}_{h}^{s}(Q) \oplus \nabla U_{h}(Q) \supset \boldsymbol{\Psi}_{h}(Q)
$$

and

$$
\operatorname{dim} \mathbf{V}_{h}^{s}(Q)=k^{2}, \operatorname{dim} \mathbf{\Psi}_{h}=2 k(k+1)-1, \operatorname{dim} U_{h}(Q)=(k+1)^{2}-1 .
$$

So (10) is satisfied. This completes the verification of $(\mathbf{H} 4)$.

Therefore we can define a new mixed finite volume scheme using the spaces $\mathbf{V}_{h}(Q)$ and $N_{h}(Q)$ for the vector part and the corresponding pressure part, respectively. To obtain the solution $\left(\mathbf{u}_{h}, p_{h}\right)$ of higher order mixed volume methods, it suffices to solve the equivalent system (15) using the nonconforming space $N_{h}(Q)$.

\section{Acknowledgements}

This paper has been supported by 2014 Hannam University Research Fund. 


\section{References}

[1] P. A. Raviart, J. M. Thomas, A mixed finite element method for 2nd order elliptic problems, Mathematical aspects of finite element methods, Lecture Notes in Math., Springer, Berlin (1977).

[2] F. Brezzi, J. Douglas, Jr., and L. D. Marini, Two families of mixed finite elements for second order elliptic problems, Numer. Math., 47, (1985), 217-235, doi:10.1007/BF01389710.

[3] D. N. Arnold, F. Brezzi, Mixed and nonconforming finite element methods: Implementation, postprocessing and error estimates, RAIRO Model. Math. Anal. Numer. , 19, (1985), 7-32.

[4] F. Brezzi, J. Douglas, M. Fortin et al., Efficient rectangular mixed finite elements in two and three variables, RAIRO Model. Math. Anal. Numer. , 21, (1987), 581-604.

[5] Z. Chen, Analysis of mixed methods using conforming and nonconforming finite element methods, RAIRO Model. Math. Anal. Numer. , 27, (1993), $9-34$.

[6] T. Arbogast, Z. Chen, On the implementation of mixed methods as nonconforming methods for second order elliptic problems, Math. Comp., 64, (1995), 943-973.

[7] L. D. Marini, An inexpensive method for the evaluation of the solution of the lowest order Raviart-Thomas mixed method, SIAM J. Numer. Anal., 22, (1995), 493-496, doi:10.1137/0722029.

[8] Z. Chen, Equivalence between and multigrid algorithms for nonconforming and mixed methods for second-order elliptic problems, East-West J. Numer. Math. , 4, (1996), 1-33.

[9] J. P. Croisille, I. Greff, Some nonconforming mixed box schemes for elliptic problems, Numer. Methods Partial Differential Equations , 18, (2002), 355373.

[10] S. H. Chou, D. Y. Kwak, K. Y. Kim, Mixed finite volume methods on nonstaggered quadrilateral grids for elliptic problems, Math. Comp., 72, (2002), 525-539.

[11] I. Greff, Nonconforming box-schemes for elliptic problems on rectangular grids, SIAM J. Numer. Anal. , 45, (2007), 946-968, doi:10.1137/050647578. 
[12] D. Y. Kwak, A new class of higher order mixed finite volume methods for elliptic problems, SIAM J. Numer. Anal., 50, (2012), 1941-1598, doi:10.1137/100812446. 